\title{
ON THE EXACT WKB ANALYSIS FOR THE THIRD ORDER ORDINARY DIFFERENTIAL EQUATIONS WITH A LARGE PARAMETER*
}

\author{
TAKASHI AOKI ${ }^{\dagger}$, TAKAHIRO KAWAI $^{\ddagger}$, AND YOSHITSUGU TAKEI ${ }^{\S}$
}

0. Introduction. Exact WKB analysis of linear ordinary differential equations of the $n$-th order with $n \gtreqless 2$ has not yet been well established; its local aspect is clarified under the assumption that the discriminant of the characteristic equation is of simple zeros ([AKT, Theorem 1.4 and Theorem 1.6]), but its global aspect is far from complete understanding ([BNR], [AKT, Section 2]). The purpose of this paper is to examine the validity of our Ansatz concerning the Stokes geometry ([AKT, Section 2]) through the study of particular differential equations whose solutions can be explicitly given in the form of integrals.

A class of differential equations of the third order which admits the integral representation of its solutions is analytically studied in Section 1. Note that this class of differential equations originated from some concrete quantum-mechanical problems $([\mathrm{CH}])$. We hope the equations of this class will play an important role as a kind of the "model equation" in our future study of exact WKB analysis of higher order equations.

As the integral representation of a solution is closely related to the Borel sum of the WKB solutions ([U1], [U2], [H]), we then do in Section 2 a computer-assisted study of the integral to see how the configuration of the saddle points and the steepest descent paths is changed as the variable of the integral moves around. This approach is due to Uchiyama ([U1], [U2]), and it is also related to the hyperasymptotic analysis due to Berry and Howls ([BH] $)$. In a word, our Ansatz is validated in all the examples we have studied.

For the convenience of the reader, here we present the Ansatz given in [AKT] in a slightly more precise and general form. We basically follow [AKT] in the usage of notions and notations such as the notion "a new turning point" and the symbol " $0<1$ " attached to a Stokes curve. (In this paper we use the naming "a Stokes curve" rather than "a Stokes line" following recent literature.) Here we note that a new turning point is determined in terms of a self-intersection point of a bicharacteristic curve associated with the operator in question. (See Section 1.2 below for the precise definition.) We also note that the symbol " $0<1$ " indicates the dominance of the WKB solution $\psi_{1}$ over $\psi_{0}$ along the Stokes curve with the symbol attached.

Now we propose the following Ansatz concerning the Stokes geometry for an operator

$$
H=\frac{d^{3}}{d x^{3}}+3 \eta^{2} a(x) \frac{d}{d x}+\eta^{3} b(x)
$$

\footnotetext{
*Received October 16, 1998; accepted for publication January 5, 1999.

† Department of Mathematics and Physics, Faculty of Science and Technology, Kinki University, Higashi-Osaka, 577-8502 Japan (aoki@math.kindai.ac.jp). Supported in part by Grant-in-Aid for Scientific Research (C) (09640234), Japanese Ministry of Education, Science, Sports and Culture.

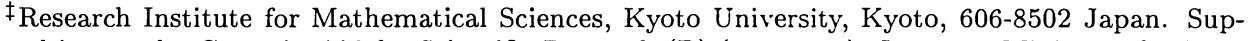
ported in part by Grant-in-Aid for Scientific Research (B) (08454029), Japanese Ministry of Education, Science, Sports and Culture.

$\S$ Research Institute for Mathematical Sciences, Kyoto University, Kyoto, 606-8502 Japan (takei@kurims.kyoto-u.ac.jp). Supported in part by Grant-in-Aid for Encouragement of Young Scientists (09740101), Japanese Ministry of Education, Science, Sports and Culture.
} 
where $\eta$ is a large parameter and $a(x)$ and $b(x)$ are polynomials satisfying

$$
6 a^{2} \frac{d a}{d x}+b \frac{d b}{d x} \neq 0 \text { if } 4 a^{3}+b^{2} \text { vanishes. }
$$

Note that the condition (0.2) (the simple discriminant condition) guarantees that the Borel transform $\hat{H}$ of $H$, i.e., the partial differential operator

$$
\frac{\partial^{3}}{\partial x^{3}}+3 a(x) \frac{\partial^{3}}{\partial x \partial y^{2}}+b(x) \frac{\partial^{3}}{\partial y^{3}}
$$

is of simple characteristics on $\left\{(x, y ; \xi, \eta) \in T^{*} \mathbb{C}^{2} ; \eta \neq 0\right\}$. In concrete examples we sometimes deal with operators of the form $d^{3} / d x^{3}+3 \eta^{2}\left(a_{0}(x)+a_{1}(x) \eta^{-1}+\right.$ $\left.a_{2}(x) \eta^{-2}\right) d / d x+\eta^{3}\left(b_{0}(x)+b_{1}(x) \eta^{-1}+b_{2}(x) \eta^{-2}+b_{3}(x) \eta^{-3}\right)$, but the contents of the Ansatz below is not affected by the regular perturbation terms such as $3 a_{1}(x) \eta \cdot d / d x$ etc, as the Stokes geometry concerns only with the principal part of the operator $H$.

Ansatz. (Cf. [AKT, Section 2].)

Assume that the Riemann surface $\mathcal{R}=\left\{(x, \xi) ; \xi^{3}+3 a(x) \xi+b(x)=0\right\}$ is connected. Suppose further the period integral $\oint_{\gamma} \xi(x) d x$ for a closed path $\gamma$ in $\mathcal{R}$ should never be a real number. To find the Stokes geometry for the operator $H$ we proceed as follows:

(i) Draw Stokes curves emanating from ordinary turning points of the operator $H$.

(ii) Let $T$ be a new turning point and let $X$ be the corresponding self-intersection point of a bicharacteristic curve for $\hat{H}$ (i.e., $T$ is the $x$-component of the coordinate $(x, y)$ of the point $X)$. It then follows from the definition that two bicharacteristic curves $b_{1}$ and $b_{2}$ pass through the point $X$ and that $-d y / d x$ evaluated along $b_{1}$ (resp., $b_{2}$ ) coincides with a characteristic root $\xi_{j}(x)$ (resp., $\xi_{k}(x)$ ), i.e., a solution of the equation

$$
\xi^{3}+3 a(x) \xi+b(x)=0 .
$$

We then draw the Stokes curve $(j, k)$ passing through $T$, that is, we consider the one-dimensional curve

$$
\operatorname{Im} \int_{T}^{x}\left(\xi_{j}(x)-\xi_{k}(x)\right) d x=0
$$

The portion of this Stokes curve where Re $\int_{T}^{x}\left(\xi_{j}(x)-\xi_{k}(x)\right) d x>0$ (resp., $\operatorname{Re} \int_{T}^{x}\left(\xi_{j}(x)\right.$ $\left.-\xi_{k}(x)\right) d x<0$ ) is labeled as $j>k$ (resp., $j<k$ ).

(iii) A Stokes curve considered in (ii) should be ignored in the Stokes geometry until it passes through the ordered crossing point in the sense of [BNR] of Stokes curves considered either in (i) or in (ii).

(iv) If a Stokes curve considered in (ii) reaches an ordered crossing point $A$ of Stokes curves considered either in (i) or in (ii), then we include in the Stokes geometry the portion of the curve (0.5) after it passes through the point $A$. In particular, if a Stokes curve considered in (ii) never reaches an ordered crossing point of Stokes curves, it is ignored in the Stokes geometry.

(v) The Stokes curves formed in (iv) together with those in (i) describe the Stokes geometry. 
(vi) If three of the Stokes curves in (v) meet at a point $B$, then we have the following rule for the coefficients of the connection of WKB solutions normalized by choosing a (generic) point $x_{0}$ near $B$ as the end-point of their integration.

RulE. Choose a pair of Stokes curves which form an ordered crossing at $B$. Then the connection coefficients associated with these two Stokes curves should be kept intact near $B$, and the connection coefficient of the other Stokes curve is changed abruptly at $B$ so that

$$
\gamma+\gamma^{\prime}=\alpha \beta
$$

holds, where $\alpha, \beta, \gamma$ and $\gamma^{\prime}$ are the connection coefficients as we cross a Stokes curve in the direction designated by the symbol $\rightarrow$ in Figure 1.

(i)

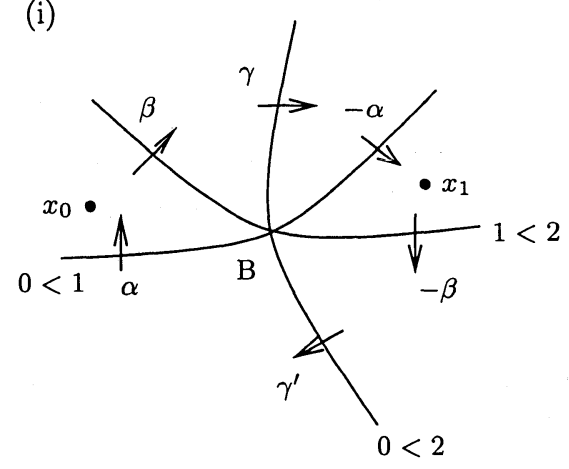

(ii)

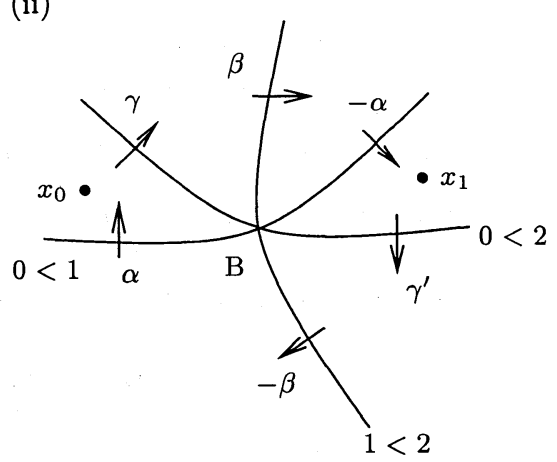

Figure 1

REMARK 0.1. The above Rule is based on the following observation due to [BNR]. (See also [AKT, Section 2].) Let us consider (in either configuration (i) or configuration (ii)) the analytic continuation of a WKB solution $\psi_{2}$ from the point $x_{0}$ to the point $x_{1}$ following the path which turns around $B$ in a clockwise manner. Then the resulting function near $x_{1}$ is

$$
\psi_{2}+\beta \psi_{1}+\gamma \psi_{0}-\beta \alpha \psi_{0}
$$

If we do a similar continuation turning around $B$ in an anti-clockwise manner, we will find

$$
\psi_{2}-\gamma^{\prime} \psi_{0}+\beta \psi_{1}
$$

Since the operator $H$ has no singular points near $B$, these two functions should coincide, leading to the relation (0.6).

We note that the above Rule is thus of local character, and hence the global self-consistency of the above Rule is an open problem. We also note that the above Rule sometimes makes vanish the connection coefficient attached to some portion of a Stokes curve. In such a circumstance we ignore the portion of a Stokes curve in question in the final form of the Stokes geometry.

REMARK 0.2. The same reasoning as above applied to a neighborhood of a new turning point explains why no connection phenomena occur there though the confluence of two singularities of the Borel transform of WKB solutions occurs in general (as 
the general theory on linear partial differential equations (see, e.g. [SKK, Chap. II]) tells). In fact, a solution $\hat{\psi}$ of the equation $\hat{H} \hat{\psi}=0$ has singularities at $\left(x, y_{j}(x)\right)$ and $\left(x, y_{k}(x)\right)$ in general. Since $d y_{j} / d x=-\xi_{j}(x) \neq-\xi_{k}(x)=d y_{k} / d x$ at $T, \operatorname{Re} y_{j}-\operatorname{Re} y_{k}$ changes its sign at $T$ along the curve (0.5). Hence the analytic continuation of $\psi_{j}$ across the portion of the curve where $\operatorname{Re} y_{j}>\operatorname{Re} y_{k}$ remains unchanged, while that across the portion where $\operatorname{Re} y_{k}>\operatorname{Re} y_{j}$ is of the form $\psi_{j}+\gamma \psi_{k}$ for a constant $\gamma$. Thus $\gamma$ should vanish. Otherwise stated, no connection occurs near $T$. Thus the point $B$ in (vi) happens to be the point $A$ in (iv), then either $\gamma$ or $\gamma^{\prime}$. should be 0 in (0.6). Essentially the same argument was also used by Voros ([V, p. 244]) in establishing the connection formula for the second order differential equations.

REMARK 0.3. The connectedness assumption on the Riemann surface is to avoid degenerate cases, and the reality assumption on the period is to determine without ambiguities the dominance relation among WKB solutions; since the Borel transform of a WKB solution has a singularity shifted by a period (cf. [V], [DDP]) in general and since the dominance relation is determined by the comparison of the real part of the singularities of the Borel transform of WKB solutions, we need this assumption.

\section{Some analytic properties of the Carroll-Hioe equation.}

1.1. Integral representation of a solution. In discussing the three-level Landau-Zener model, Carroll and Hioe ([CH, p. 2068]) encountered the following equation (CHO), where $r_{j}(j=1,2,3)$ and $\Omega_{12}$ and $\Omega_{23}$ are some real constants.

(CHO)

$$
\begin{aligned}
& \frac{d^{3} \psi}{d x^{3}}+2 i\left(r_{1}+r_{2}+r_{3}\right) x \frac{d^{2} \psi}{d x^{2}}+\left\{\left(-4\left(r_{1} r_{2}+r_{2} r_{3}\right.\right.\right. \\
& \left.\left.\quad+r_{3} r_{1}\right) x^{2}+2 i\left(2 r_{1}+r_{2}\right)+\frac{1}{4}\left[\left(\Omega_{12}\right)^{2}+\left(\Omega_{23}\right)^{2}\right]\right\} \frac{d \psi}{d x} \\
& \quad+\left\{-8 i r_{1} r_{2} r_{3} x^{3}-4 r_{1}\left(2 r_{2}+r_{3}\right) x\right. \\
& \left.\quad+\frac{i}{2}\left[\left(\Omega_{12}\right)^{2} r_{3}+\left(\Omega_{23}\right)^{2} r_{1}\right] x\right\} \psi=0 .
\end{aligned}
$$

They ingeniously observed that the singular coordinate transformation

$$
z=x^{2}
$$

brings $(\mathrm{CHO})$ to the following equation:

$$
\begin{aligned}
& z \frac{d^{3} \psi}{d z^{3}}+\left[i\left(r_{1}+r_{2}+r_{3}\right) z+\frac{3}{2}\right] \frac{d^{2} \psi}{d z^{2}}+\left\{-\left(r_{1} r_{2}+r_{2} r_{3}\right.\right. \\
& \left.\left.\quad+r_{3} r_{1}\right) z+\frac{i}{2}\left(3 r_{1}+2 r_{2}+r_{3}\right)+\frac{1}{16}\left[\left(\Omega_{12}\right)^{2}+\left(\Omega_{23}\right)^{2}\right]\right\} \frac{d \psi}{d z} \\
& \quad+\left\{-i r_{1} r_{2} r_{3} z-\frac{1}{2} r_{1}\left(2 r_{2}+r_{3}\right)+\frac{i}{16}\left[\left(\Omega_{12}\right)^{2} r_{3}+\left(\Omega_{23}\right)^{2} r_{1}\right]\right\} \psi=0 .
\end{aligned}
$$

An important feature of the equation (1.2) is that each coefficient is linear in $z$; hence the Laplace method gives an integral representation of a solution (1.2) in the form

$$
\psi(z)=\int\left(\zeta+i r_{1}\right)^{-1+i p}\left(\zeta+i r_{2}\right)^{-\frac{1}{2}-i p+i q}\left(\zeta+i r_{3}\right)^{-i q} \exp (\zeta z) d \zeta
$$

with

$$
p=\frac{\left(\Omega_{12}\right)^{2}}{16\left(r_{1}-r_{2}\right)} \quad \text { and } \quad q=\frac{\left(\Omega_{23}\right)^{2}}{16\left(r_{2}-r_{3}\right)}
$$


If we assume $r_{j}$ 's and $\Omega_{j k}$ 's contain a large parameter $\eta$ linearly, then, with the scaled constants being denoted by the same symbol, we find an equation with a large parameter $\eta$ :

$$
\begin{aligned}
& \frac{d^{3} \psi}{d x^{3}}+2 i\left(r_{1}+r_{2}+r_{3}\right) \eta x \frac{d^{2} \psi}{d x^{2}}+\left\{\left(-4\left(r_{1} r_{2}+r_{2} r_{3}\right.\right.\right. \\
& \left.\left.\quad+r_{3} r_{1}\right) \eta^{2} x^{2}+2 i\left(2 r_{1}+r_{2}\right) \eta+\frac{1}{4}\left[\left(\Omega_{12}\right)^{2}+\left(\Omega_{23}\right)^{2}\right] \eta^{2}\right\} \frac{d \psi}{d x} \\
& \quad+\left\{-8 i r_{1} r_{2} r_{3} \eta^{3} x^{3}-4 r_{1}\left(2 r_{2}+r_{3}\right) \eta^{2} x\right. \\
& \left.\quad+\frac{i}{2}\left[\left(\Omega_{12}\right)^{2} r_{3}+\left(\Omega_{23}\right)^{2} r_{1}\right] \eta^{3} x\right\} \psi=0 .
\end{aligned}
$$

In what follows we allow $r_{j}$ 's and $\Omega_{j k}$ 's in $(\mathrm{CH})$ to be complex, forgetting the physical origin of the equation. Furthermore, even if we replace $\left(2 r_{1}+r_{2}\right) \eta$ and $r_{1}\left(2 r_{2}+r_{3}\right) \eta^{2} x$ in $(\mathrm{CH})$ respectively by $c_{1} \eta$ and $c_{2} \eta^{2} x$ with $c_{1}$ and $c_{2}$ being arbitrary constants (i.e., unrelated to $r_{j}$ 's), all the arguments we will give below go equally well. Hence in what follows we discuss the following generalized Carroll-Hioe equation (GCH), rather than $(\mathrm{CH})$ itself:

$(\mathrm{GCH})$

$$
\begin{aligned}
& \frac{d^{3} \psi}{d x^{3}}+2 i\left(r_{1}+r_{2}+r_{3}\right) \eta x \frac{d^{2} \psi}{d x^{2}}+\left\{\left(-4\left(r_{1} r_{2}+r_{2} r_{3}\right.\right.\right. \\
& \left.\left.\quad+r_{3} r_{1}\right) \eta^{2} x^{2}+2 i c_{1} \eta+\frac{1}{4}\left[\left(\Omega_{12}\right)^{2}+\left(\Omega_{23}\right)^{2}\right] \eta^{2}\right\} \frac{d \psi}{d x} \\
& \quad+\left\{-8 i r_{1} r_{2} r_{3} \eta^{3} x^{3}-4 c_{2} \eta^{2} x\right. \\
& \left.\quad+\frac{i}{2}\left[\left(\Omega_{12}\right)^{2} r_{3}+\left(\Omega_{23}\right)^{2} r_{1}\right] \eta^{3} x\right\} \psi=0 .
\end{aligned}
$$

In accordance with the introduction of a large parameter $\eta$ into the differential equation, the integral representation (1.3) may be replaced (by disposing of some factor depending only on $\eta$ ) with

$$
\psi(z)=\int a(\zeta) \exp (\eta \phi(\zeta, z)) d \zeta
$$

where

$$
a(\zeta)=\prod_{j=1}^{3}\left(\zeta+i r_{j}\right)^{\mu_{j}}
$$

with

$$
\mu_{1}=\frac{\frac{3}{2} r_{1}^{2}-\tilde{c}_{1} r_{1}+\tilde{c}_{2}}{\left(r_{2}-r_{1}\right)\left(r_{3}-r_{1}\right)}-1
$$

$$
\mu_{2}=\frac{\frac{3}{2} r_{2}^{2}-\tilde{c}_{1} r_{2}+\tilde{c}_{2}}{\left(r_{1}-r_{2}\right)\left(r_{3}-r_{2}\right)}-1
$$

$$
\mu_{3}=\frac{\frac{3}{2} r_{3}^{2}-\tilde{c}_{1} r_{3}+\tilde{c}_{2}}{\left(r_{1}-r_{3}\right)\left(r_{2}-r_{3}\right)}-1
$$


and

$$
\phi(\zeta, z)=\zeta z+i p \log \left(\zeta+i r_{1}\right)+(-i p+i q) \log \left(\zeta+i r_{2}\right)-i q \log \left(\zeta+i r_{3}\right) .
$$

Here we used the symbols $\tilde{c}_{1}$ and $\tilde{c}_{2}$ respectively to denote $\left(c_{1}+r_{1}+r_{2}+r_{3}\right) / 2$ and $c_{2} / 2$, and the constants $p$ and $q$ have the same expression as in (1.4). We note that $\mu_{j}$ 's given above satisfy the following relation:

$$
\mu_{1}+\mu_{2}+\mu_{3}=-3 / 2 \text {. }
$$

The saddle points of the integral (1.5), i.e., the points where $\partial \phi / \partial \zeta$ vanishes, are given by the following equation:

$$
z+\frac{i p}{\zeta+i r_{1}}+\frac{-i p+i q}{\zeta+i r_{2}}+\frac{-i q}{\zeta+i r_{3}}=0
$$

Let $\zeta_{j}(z)(j=0,1,2)$ be the solutions of $(1.10)$ and endow the precise meaning of the integral (1.5) by choosing a steepest descent path $C_{j}$, i.e., an integral curve of the vector field $\nabla_{\zeta} \operatorname{Re} \phi(\zeta, z)$, passing through $\zeta_{j}(z)$. Then, aside from a multiplicative factor depending only on $\eta$, each of them asymptotically represents a WKB solution whose logarithmic derivative begins with $\phi\left(\zeta_{j}(z), z\right)$.

REMARK 1.1. When $r_{1}+r_{2}+r_{3} \neq 0$, we may replace $\psi$ by $\exp \left(\int^{x} \frac{2 i}{3} \eta\left(r_{1}+\right.\right.$ $\left.\left.r_{2}+r_{3}\right) x d x\right) \psi$ to eliminate the second order part, so that the equation takes the form discussed in Introduction. But, to keep the symmetry of the coefficients, we do not employ this replacement of the unknown function in the computation below.

1.2. Bicharacteristics of the Borel transform of (GCH). One of the most important ingredients of the exact WKB analysis is the structure of singularities of Borel-transformed WKB solutions, which propagate along the bicharacteristic curves for the Borel-transformed operator in the case of linear differential equations. A peculiar feature of the equation $(\mathrm{GCH})$ is that the bicharacteristics of its Borel transform can be described explicitly in terms of elliptic functions. In this subsection we compute the explicit description of bicharacteristics of

$$
\begin{aligned}
& \frac{\partial^{3} \hat{\psi}}{\partial x^{3}}+2 i\left(r_{1}+r_{2}+r_{3}\right) x \frac{\partial^{3} \hat{\psi}}{\partial x^{2} \partial y} \\
& +\left\{-4\left(r_{1} r_{2}+r_{2} r_{3}+r_{3} r_{1}\right) x^{2}+\frac{1}{4}\left[\left(\Omega_{12}\right)^{2}+\left(\Omega_{23}\right)^{2}\right]\right\} \frac{\partial^{3} \hat{\psi}}{\partial x \partial y^{2}} \\
& \quad+\left\{-8 i r_{1} r_{2} r_{3} x^{3}+\frac{i}{2}\left[\left(\Omega_{12}\right)^{2} r_{3}+\left(\Omega_{23}\right)^{2} r_{1}\right] x\right\} \frac{\partial^{3} \hat{\psi}}{\partial y^{3}} \\
& \quad+2 i c_{1} \frac{\partial^{2} \hat{\psi}}{\partial x \partial y}-4 c_{2} x \frac{\partial^{2} \hat{\psi}}{\partial y^{2}}=0
\end{aligned}
$$

i.e., the Borel transform (with respect to $\eta$ ) of $(\mathrm{GCH})$.

Let

$$
p(x, y, \xi, \eta)=\xi^{3}+2 \sigma_{1} x \xi^{2} \eta+4\left(\sigma_{2} x^{2}+\alpha_{1}\right) \xi \eta^{2}+8\left(\sigma_{3} x^{3}+\alpha_{0} x\right) \eta^{3}
$$

be the principal symbol of (1.11), where $\sigma_{j}$ and $\alpha_{k}$ denote the following constants respectively:

$$
\begin{aligned}
& \sigma_{1}=i\left(r_{1}+r_{2}+r_{3}\right), \quad \sigma_{2}=-\left(r_{1} r_{2}+r_{2} r_{3}+r_{3} r_{1}\right), \quad \sigma_{3}=-i r_{1} r_{2} r_{3}, \\
& \alpha_{1}=\frac{1}{16}\left[\left(\Omega_{12}\right)^{2}+\left(\Omega_{23}\right)^{2}\right], \quad \alpha_{0}=\frac{i}{16}\left[\left(\Omega_{12}\right)^{2} r_{3}+\left(\Omega_{23}\right)^{2} r_{2}\right] .
\end{aligned}
$$


Here and in what follows $\alpha_{1}$ is assumed to be a non-zero constant. We are interested in the null-bicharacteristic strip of (1.11), that is, a solution curve of the following Hamiltonian system in the cotangent space $T^{*} \mathbb{C}^{2}$ of $\mathbb{C}_{(x, y)}^{2}$ which satisfies $p(x, y, \xi, \eta)=$ 0 :

$$
\left\{\begin{array}{l}
\dot{x}=\frac{\partial p}{\partial \xi}=3 \xi^{2}+4 \sigma_{1} x \xi \eta+4\left(\sigma_{2} x^{2}+\alpha_{1}\right) \eta^{2} \\
\dot{y}=\frac{\partial p}{\partial \eta}=2 \sigma_{1} x \xi^{2}+8\left(\sigma_{2} x^{2}+\alpha_{1}\right) \xi \eta+24\left(\sigma_{3} x^{3}+\alpha_{0} x\right) \eta^{2} \\
\dot{\xi}=\frac{\partial p}{\partial x}=-\left(2 \sigma_{1} \xi^{2} \eta+8 \sigma_{2} x \xi \eta^{2}+8\left(3 \sigma_{3} x^{2}+\alpha_{0}\right) \eta^{3}\right) \\
\dot{\eta}=-\frac{\partial p}{\partial y}=0
\end{array}\right.
$$

Note that its projection to the base space $\mathbb{C}_{(x, y)}^{2}$ is, by definition, a (null-) bicharacteristic curve. Since $p(x, y, \xi, \eta)$ does not depend on $y$ in our case, we may assume without loss of generality that $\eta$ is identically equal to 1 . Furthermore the first equation together with the third one of (1.13) forms a system in involution:

$$
\left\{\begin{array}{l}
\dot{x}=3 \xi^{2}+4 \sigma_{1} x \xi+4\left(\sigma_{2} x^{2}+\alpha_{1}\right) \\
\dot{\xi}=-\left(2 \sigma_{1} \xi^{2}+8 \sigma_{2} x \xi+8\left(3 \sigma_{3} x^{2}+\alpha_{0}\right)\right),
\end{array}\right.
$$

which is also a Hamiltonian system with the Hamiltonian $\tilde{p}(x, \xi)=\left.p(x, y, \xi, \eta)\right|_{\eta \equiv 1}$. Once a solution $(x(t), \xi(t))$ of (1.14) is given, then a null-bicharacteristic strip of (1.11) can be easily obtained by integrating the second equation of (1.13).

To solve (1.14), we employ the following canonical transformation corresponding to the singular transformation (1.1):

$$
z=x^{2}, \quad \zeta=\frac{\xi}{2 x} .
$$

In the $(z, \zeta)$-variable the Hamiltonian system (1.14) becomes

$$
\left\{\begin{array}{l}
\dot{z}=8 \sqrt{z}\left(3 z \zeta^{2}+2 \sigma_{1} z \zeta+\sigma_{2} z+\alpha_{1}\right) \\
\dot{\zeta}=-8 \sqrt{z}\left(\zeta^{3}+\sigma_{1} \zeta^{2}+\sigma_{2} \zeta+\sigma_{3}\right)
\end{array}\right.
$$

with $q(z, \zeta) \stackrel{\text { def }}{=} z \zeta^{3}+\sigma_{1} z \zeta^{2}+\left(\sigma_{2} z+\alpha_{1}\right) \zeta+\sigma_{3} z+\alpha_{0}=0$. In particular, it follows from the relation $q(z, \zeta)=0$ that

$$
z=-\frac{\alpha_{1} \zeta+\alpha_{0}}{R(\zeta)}
$$

holds, where

$$
R(\zeta) \stackrel{\text { def }}{=} \zeta^{3}+\sigma_{1} \zeta^{2}+\sigma_{2} \zeta+\sigma_{3}=\left(\zeta+i r_{1}\right)\left(\zeta+i r_{2}\right)\left(\zeta+i r_{3}\right)
$$

Furthermore, substitution of (1.17) into (1.16) entails the following differential equation for $\zeta$ :

$$
\begin{aligned}
\dot{\zeta}^{2} & =-64\left(\alpha_{1} \zeta+\alpha_{0}\right) R(\zeta) \\
& =-64\left(\alpha_{1} \zeta+\alpha_{0}\right)\left(\zeta+i r_{1}\right)\left(\zeta+i r_{2}\right)\left(\zeta+i r_{3}\right)
\end{aligned}
$$


Thus $\zeta=\zeta(t)$ is an elliptic function defined as an inverse function of the elliptic integral

$$
t=\int_{\zeta_{0}}^{\zeta} \frac{d \zeta}{8 i\left\{\left(\alpha_{1} \zeta+\alpha_{0}\right) R(\zeta)\right\}^{1 / 2}}
$$

where $\zeta_{0}$ is a fixed point which should be determined by the initial condition. Consequently a solution of the system (1.14) can be expressed in the following manner:

$$
\left\{\begin{array}{l}
x(t)=\{z(t)\}^{1 / 2}=\left(-\frac{\alpha_{1} \zeta(t)+\alpha_{0}}{R(\zeta(t))}\right)^{1 / 2} \\
\xi(t)=2 \zeta(t) x(t)=2 \zeta(t)\left(-\frac{\alpha_{1} \zeta(t)+\alpha_{0}}{R(\zeta(t))}\right)^{1 / 2}
\end{array}\right.
$$

Regarding $\zeta$ as the independent variable that replaces $t$, we can also obtain the explicit description for $y=y(\zeta)$ of the system (1.13). As a matter of fact, since

$$
\begin{aligned}
\frac{d y}{d \zeta} & =\frac{\dot{y}}{\dot{\zeta}} \\
& =-\frac{1}{R(\zeta)}\left(\sigma_{1} z \zeta^{2}+2\left(\sigma_{2} z+\alpha_{1}\right) \zeta+3\left(\sigma_{3} z+\alpha_{0}\right)\right) \\
& =-\frac{1}{R(\zeta)^{2}}\left(\alpha_{1} \zeta+\alpha_{0}\right)\left(\sigma_{1} \zeta^{2}+2 \sigma_{2} \zeta+3 \sigma_{3}\right)-\frac{1}{R(\zeta)}\left(2 \alpha_{1} \zeta+3 \alpha_{0}\right) \\
& =\sum_{j=1}^{3} \beta_{j}\left(\frac{-i r_{j}}{\left(\zeta+i r_{j}\right)^{2}}+\frac{1}{\zeta+i r_{j}}\right)
\end{aligned}
$$

with

$$
\beta_{j}=\operatorname{Res}_{\zeta=-i r_{j}}\left(-\frac{\alpha_{1} \zeta+\alpha_{0}}{R(\zeta)}\right)=\frac{\alpha_{1}\left(-i r_{j}\right)+\alpha_{0}}{\left(r_{k}-r_{j}\right)\left(r_{l}-r_{j}\right)},
$$

where the indices $\{k, l\}$ are chosen so that the set $\{j, k, l\}$ coincides with $\{1,2,3\}$, we find

$$
\begin{aligned}
y(\zeta) & =\sum_{j=1}^{3} \beta_{j}\left(\frac{i r_{j}}{\zeta+i r_{j}}+\log \left(\zeta+i r_{j}\right)\right)+\text { const } \\
& =\sum_{j=1}^{3} \beta_{j}\left(\frac{i r_{j}}{\zeta+i r_{j}}+\log \left(1+\frac{i r_{j}}{\zeta}\right)\right)+\text { const } .
\end{aligned}
$$

Note that $y=y(\zeta)$ as well as $d y / d \zeta$ is singular only at $\zeta=-i r_{j}(j=1,2,3)$ and holomorphic anywhere else (including $\zeta=\infty$ ). The formulas (1.19) and (1.21) explicitly describe the null-bicharacteristic strip of (1.11) in terms of the elliptic function $\zeta=\zeta(t)$.

Using this expression of bicharacteristics, we can prove the following Proposition.

Proposition 1.1. Suppose that $\alpha_{1} \neq 0$. Then $x=0$ is a new turning point of $(G C H)$, that is, $x=0$ is the $x$-component of a self-intersection point of a (null-) bicharacteristic curve $(x(t), y(t))$ of (1.11).

Proof. The characteristic roots at $x=0$ are given by $\xi=0, \pm 2 \sqrt{\alpha_{1}} i$. In particular,

$$
\tilde{p}\left(0, \pm 2 \sqrt{\alpha_{1}} i\right)=\left.p(x, y, \xi, \eta)\right|_{x=0, \xi= \pm 2 \sqrt{\alpha_{1}} i, \eta=1}=0
$$


holds. Let us first consider two solutions of (1.14) satisfying the initial condition $\left(x_{0}, \xi_{0}\right)=\left(0, \pm 2 \sqrt{\alpha_{1}} i\right)$. In the $(z, \zeta)$-variable such solutions correspond to the following: Since $\zeta=\zeta(t)$ is an elliptic function of order 2, there exist two points $t=t_{ \pm}\left(t_{+} \neq t_{-}\right)$where $\zeta(t)$ has a simple pole with the residue $\pm \frac{1}{8 \sqrt{\alpha_{1}} i}$ respectively. Then the above solutions correspond to the solutions $(z, \zeta)=\left(z\left(t+t_{ \pm}\right), \zeta\left(t+t_{ \pm}\right)\right)$. Otherwise stated, they represent the same solution $(z(t), \zeta(t))$ which actually passes through $(x, \xi)=\left(0, \pm 2 \sqrt{\alpha_{1}} i\right)$ at $t=t_{ \pm}$respectively.

Let us next take a path $\Gamma$ in the $\zeta$-plane which goes from $\zeta=\infty$ to a point $\zeta_{0}$ near $-\alpha_{0} / \alpha_{1}$, turns around $\zeta=-\alpha_{0} / \alpha_{1}$ once and returns again to $\zeta=\infty$ with staying away from $\zeta=-i r_{j}(j=1,2,3)$ all the way. Under the mapping $\zeta=\zeta(t)$ such a path $\Gamma$ corresponds to a path $\tilde{\Gamma}$ in the $t$-plane which connects the two points $t=t_{ \pm}$. Since there is no singular point of $d y / d \zeta$ (or $\dot{y}=d y / d t$ ) on $\Gamma$ (or rather on $\tilde{\Gamma}$ ), we can readily verify

$$
\left.y\right|_{t=t_{+}}-\left.y\right|_{t=t_{-}}=\int_{\tilde{\Gamma}} \dot{y} d t=\int_{\Gamma} \frac{d y}{d \zeta} d \zeta=0 .
$$

That is, $y\left(t_{+}\right)=y\left(t_{-}\right)$holds. On the other hand we have $x\left(t_{+}\right)=x\left(t_{-}\right)=0$ as was observed earlier. This completes the proof of the Proposition.

2. Examples. In Introduction we presented our Ansatz for the Stokes geometry of higher-order ordinary differential equations. In this section we confirm the validity of our Ansatz for the (generalized) Carroll-Hioe equations through the study of integral representation of its solutions. We note that no confirmation of this sort has yet been done except for the equation discussed in [BNR] (cf. [BNR], [U1]). As we show below, the validity of our Ansatz is confirmed at several delicate points; we hope the discussion below will considerably enhance the reader's belief in our Ansatz.

All numerical computations (including drawing several pictures) in this section have been done by using Mathematica 3.0.1.

EXAMPLE 2.1. Let us consider the equation $(\mathrm{GCH})$ with the following values of parameters:

$$
\begin{aligned}
& r_{1}=-2+i, \quad r_{2}=\frac{1}{2}+2 i, \quad r_{3}=1-2 i, \\
& \Omega_{12}=-3+4 i, \quad \Omega_{23}=1-3 i, \\
& c_{1}, c_{2}: \quad \text { arbitrarily fixed constant. }
\end{aligned}
$$

This equation has six (ordinary) turning points whose approximate values are listed up below:

$$
\begin{array}{ll}
a_{1}=-0.872-0.181 i, & a_{2}=0.277-0.237 i \\
a_{3}=0.261+0.311 i, & a_{4}=-0.261-0.311 i, \\
a_{5}=-0.277+0.237 i, & a_{6}=0.872+0.181 i
\end{array}
$$

Following our Ansatz, we find the following Figure 2, which is to describe the Stokes geometry for this example. Here, and in Example 2.2 below as well, we place cuts designated by wiggly lines to fix the numbering of the characteristic roots $\left\{\xi_{j}(x)\right\}$. Note that in this example every Stokes curve asymptotically tends to infinity along one of the twelve directions and that the symbol " $j<k$ " attached to a Stokes curve depends only on this direction. (Hence in Figure 2 the symbol " $j<k$ " is attached to each direction, rather than to each Stokes curve for the sake of simplicity.) 


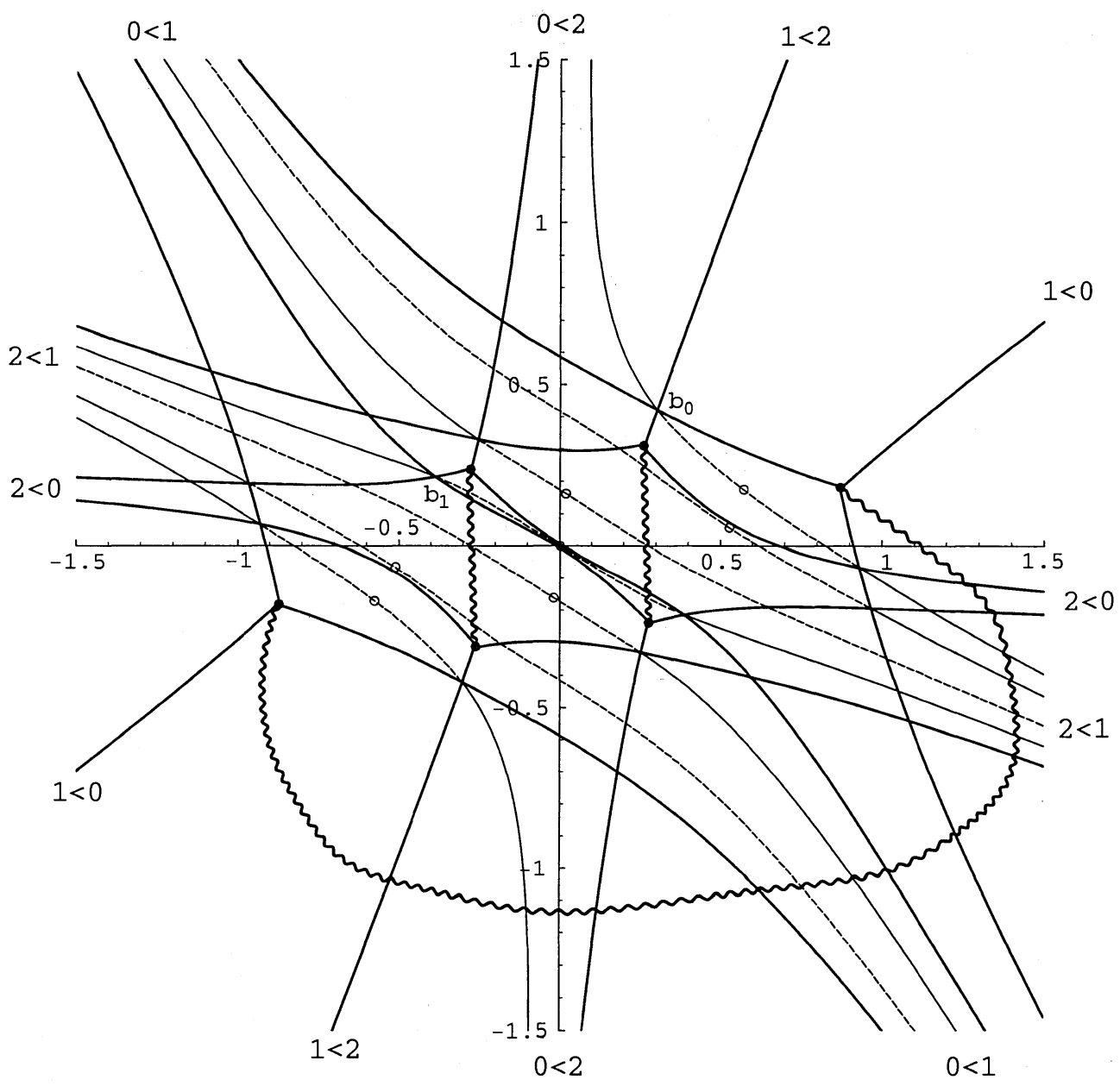

Figure 2

As is clear from Figure 2, there are seven new turning points, which are designated by "o" there (and in Example 2.2 below as well), and seven new Stokes curves emanating from them. By using the integral representation $(1.5) \sim(1.8)$ of solutions of $(\mathrm{GCH})$ we now confirm that Figure 2 gives the correct description of Stokes geometry for this example:

First let us consider the problem in a neighborhood of an ordered crossing point, say, $b_{0}$ (cf. Figure 2). As is shown in Figure 3-1 Figure 3-12, some change of configuration of saddle points $\left\{\zeta_{j}\left(x^{2}\right)\right\}$ and steepest descent paths $\left\{C_{j}\right\}$ for integral representation occurs there. (Figure 3- $j$ expresses the configuration at a point $x=\rho_{j}$. For the location of $\rho_{j}$ see Figure 3.) 


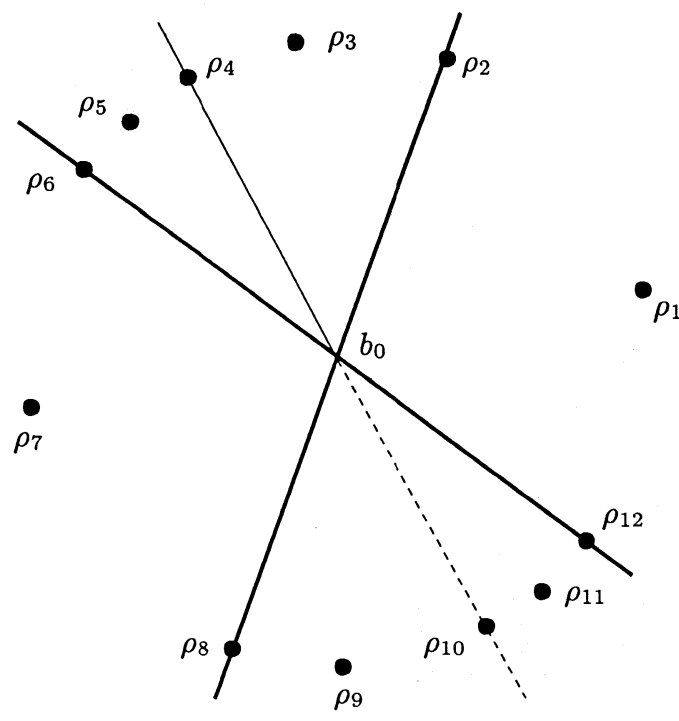

Figure 3

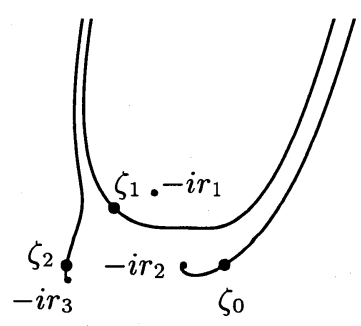

Figure 3-1

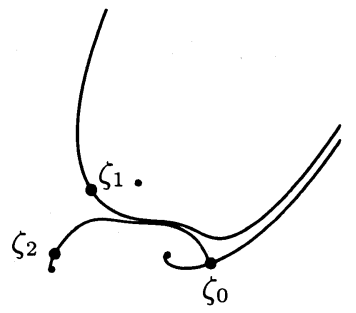

Figure 3-4

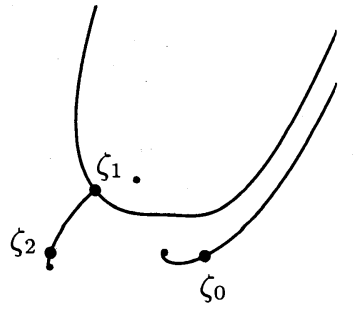

Figure 3-2

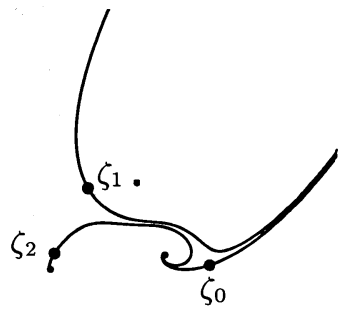

Figure 3-5

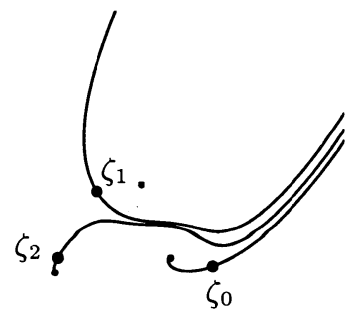

Figure 3-3

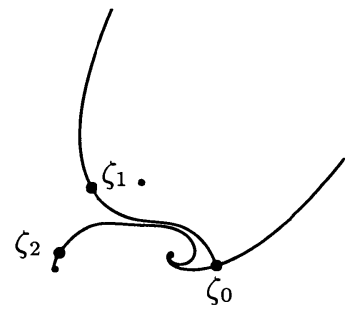

Figure 3-6 


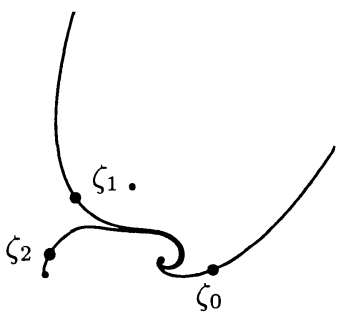

Figure 3-7

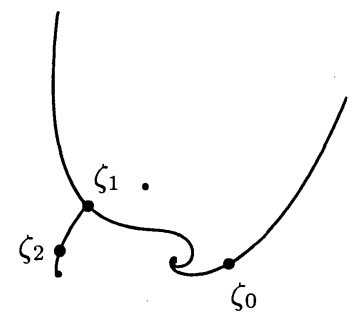

Figure 3-8

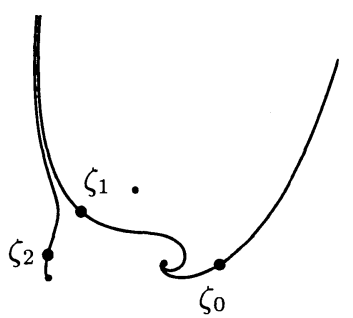

Figure 3-9

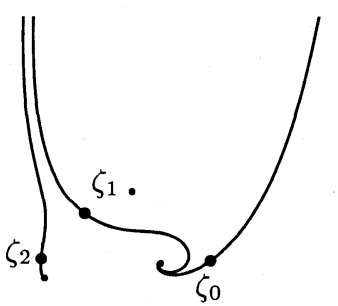

Figure 3-10

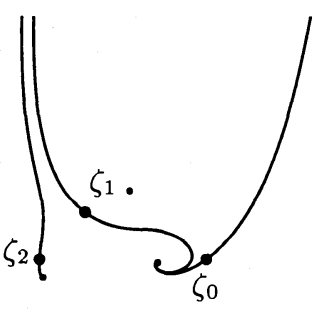

Figure 3-11

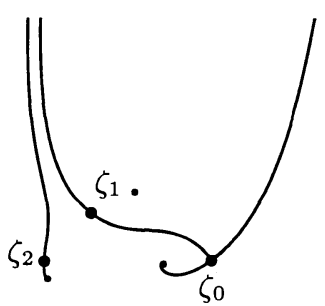

Figure 3-12

For example, at $x=\rho_{2}$ the steepest descent path $C_{2}$ flows into a saddle point $\zeta_{1}$ and consequently the configuration at $x=\rho_{1}$ is different from that at $x=\rho_{3}$ (cf. Figure 3-1 Figure 3-3). This change of configuration implies that a solution near $x=\rho_{1}$ obtained as an integral along the path $C_{2}$, which represents $(\eta$-dependent constant multiple of) a WKB solution $\psi_{2}$, should be, after analytic continuation to $x=\rho_{3}$, equal to the sum of two solutions, one of which is obtained as an integral along $C_{2}$ (representing $\psi_{2}$ ) and the other of which as an integral along $C_{1}$ (representing $\psi_{1}$ ). Otherwise stated, change of configuration of $\left\{\zeta_{j}\left(x^{2}\right)\right\}$ and $\left\{C_{j}\right\}$ for integral representation leads to a Stokes phenomenon for WKB solutions and, in particular, the change at $x=\rho_{2}$ mentioned above corresponds to the fact that $x=\rho_{2}$ is actually contained in a Stokes curve. On the other hand, such a change is not observed at $x=\rho_{10}$ (cf. Figure 3-10). This is consistent with the requirement that the portion of a (new) Stokes curve containing $x=\rho_{10}$ should be ignored (i.e., represented by a dotted line). (See [U1] also.)

In a similar manner we can confirm the validity of our Ansatz at any other points in Figure 2 except on the portion of a (new) Stokes curve containing the new turning point $x=0$ (note that the existence of a new turning point at $x=0$ is guaranteed by Proposition 1.1 in Section 1); on this portion the confirmation of the statement is somewhat delicate; let us consider an ordered crossing point $b_{1}$ in Figure 2 and look at the configuration of $\left\{\zeta_{j}\left(x^{2}\right)\right\}$ and $\left\{C_{j}\right\}$ at $x=\rho_{13}, \rho_{14}$ and $\rho_{15}$ (cf. Figure 4 and Figure 4-13 Figure 4-15). As we observe in Figure 4-14, some change of configuration occurs at $x=\rho_{14}$, although the portion of the Stokes curve in question should be ignored by our Ansatz (cf. Proposition 1.1 in Section 1 and Remark 0.2). This might sound that our Ansatz might be erroneous. However, a careful study of the structure of the integrand of the integral representation shows that there occurs a delicate cancellation among the terms that might disrupt our Ansatz near $x=\rho_{14}$. The detailed argument is as follows: If we take a solution near $x=\rho_{13}$ obtained 


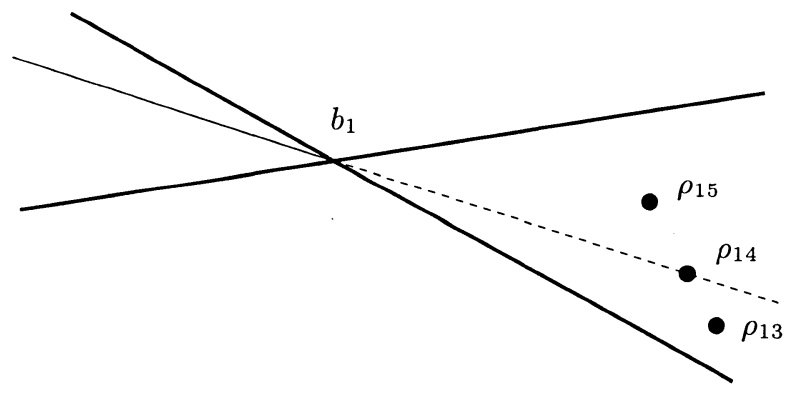

Figure 4

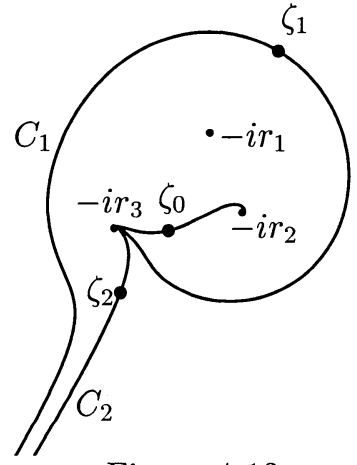

Figure 4-13

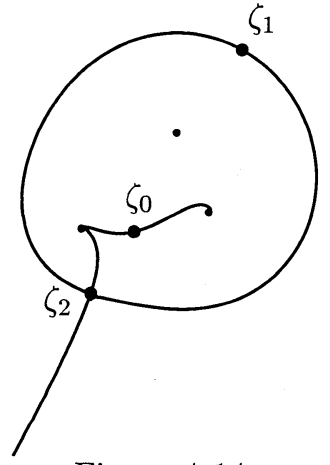

Figure 4-14

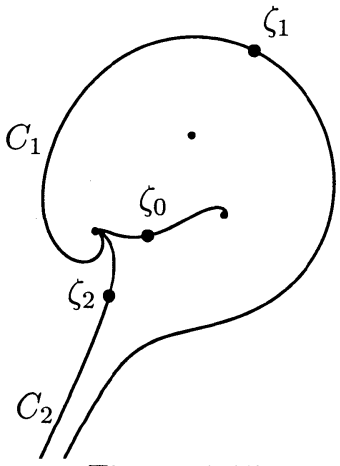

Figure 4-15

as an integral along $C_{1}$ (representing a WKB solution $\psi_{1}$ ), its analytic continuation from $x=\rho_{13}$ to $x=\rho_{15}$ picks up two solutions both of which are obtained as an integral along $C_{2}$ (representing $\psi_{2}$ ). However, the branches of $a(\zeta)$ in the integral representation (1.5) are different between these two solutions and they have opposite sign (cf. (1.9)), while the branches of $\phi(\zeta, z)$ are the same since the sum of coefficients of $\log \left(\zeta+i r_{j}\right)$ in (1.8) vanishes. Hence these two solutions picked up must cancel and no Stokes phenomenon occurs with $\psi_{1}$ at $x=\rho_{14}$. Thus our Ansatz has passed this delicate examination.

By the same method based on the computer-assisted study of integral representation of solutions we can also confirm the validity of our Ansatz for [AKT, Example 2.5].

In these examples only ordered crossing points of Stokes curves emanating from ordinary turning points ("Stokes curves considered in (i)" according to the terminology used in our Ansatz) have appeared. However, in some examples (new) Stokes curves emanating from new turning points ("Stokes curves considered in (ii)") may produce ordered crossing points. Let us consider such an example in the following:

EXAMPLE 2.2.

$$
\frac{d^{3} \psi}{d z^{3}}-3(z+2) \eta^{2} \frac{d \psi}{d z}+(4 z+6+0.3 i) \eta^{3} \psi=0
$$

This equation has three turning points $a_{0}, a_{1}$ and $a_{2}$ (cf. Figure 5). Although (2.1) is not of the form of Carroll-Hioe equation", the Laplace method again gives the following integral representation of solutions of (2.1) as all the coefficients of (2.1) are 


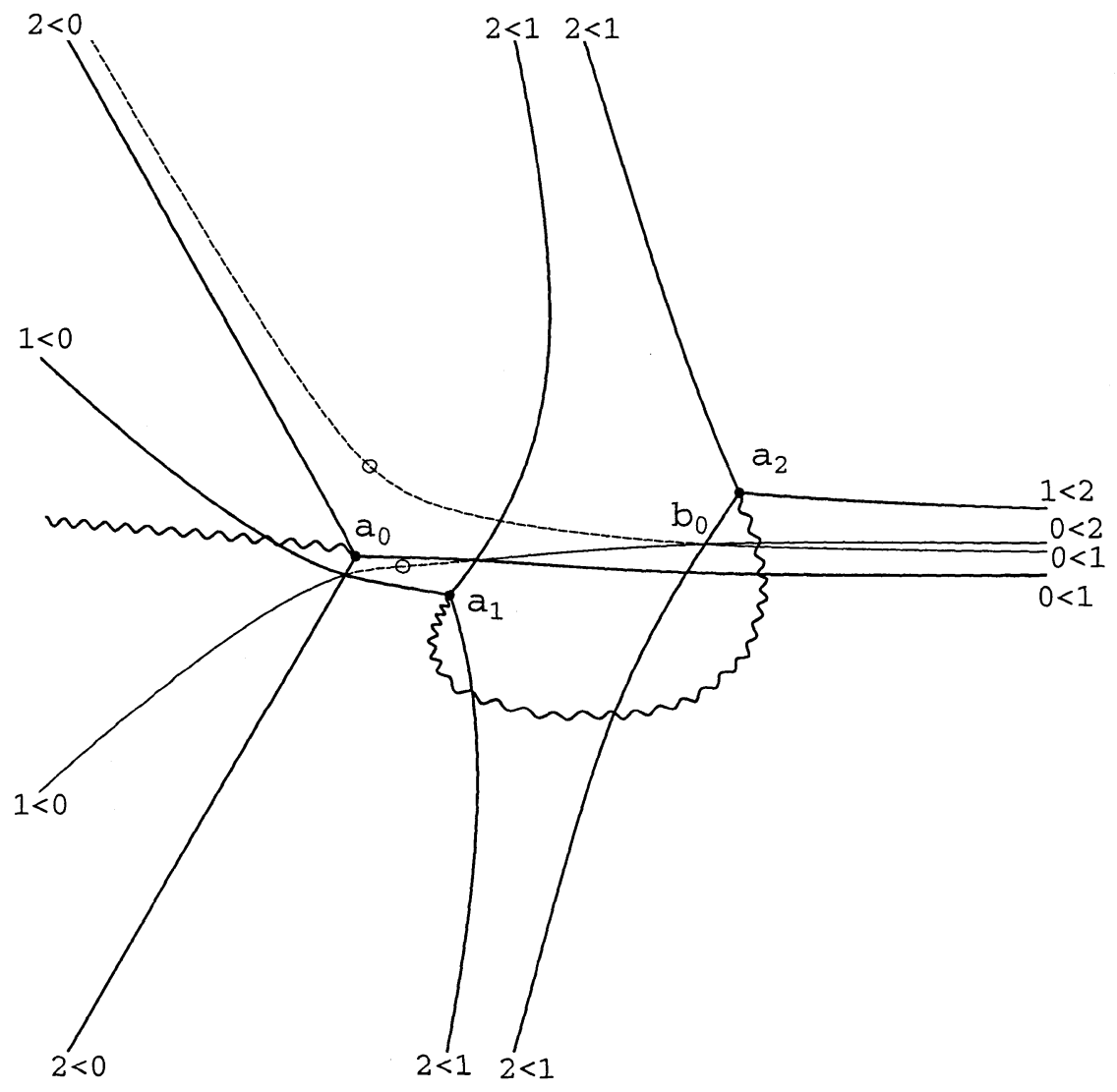

Figure 5

linear in $z$ :

$$
\psi(z)=\int a(\zeta) \exp (\eta \phi(\zeta, z)) d \zeta
$$

where

$$
a(\zeta)=\left(\zeta-\frac{4}{3}\right)^{-1}
$$

and

$$
\phi(\zeta, z)=\zeta z-\frac{1}{9} \zeta^{3}-\frac{2}{9} \zeta^{2}+\frac{38}{27} \zeta-\left(\frac{10}{81}+\frac{i}{10}\right) \log \left(\zeta-\frac{4}{3}\right)
$$

An interesting point is $z=b_{0}$, which is an ordered crossing point of an ordinary Stokes curve with a new Stokes curve. Just like Example 2.1 we have investigated the change of configuration of saddle points and steepest descent paths for (2.2) in a neighborhood of $b_{0}$ (see Figure 6 and Figure 6-1 Figure 6-12). 


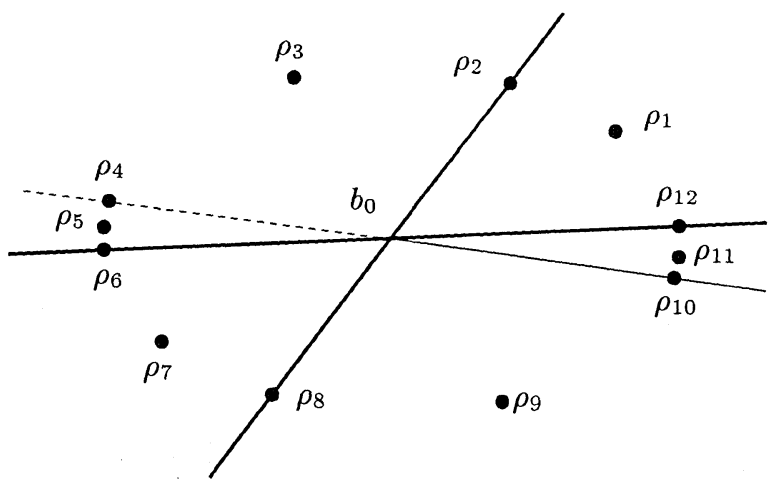

Figure 6

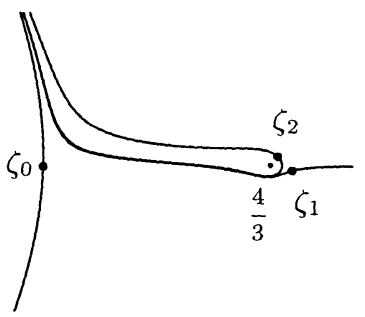

Figure 6-1

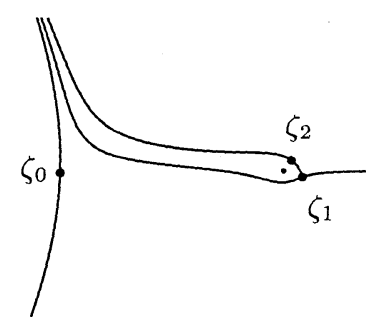

Figure 6-2

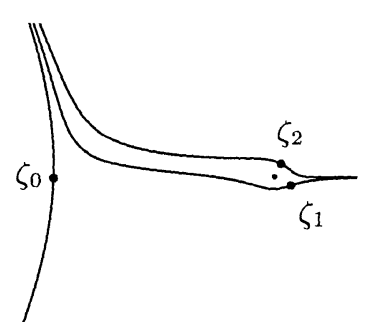

Figure 6-3

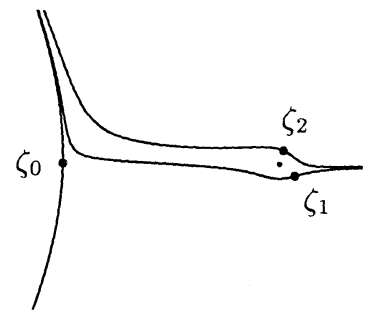

Figure 6-4

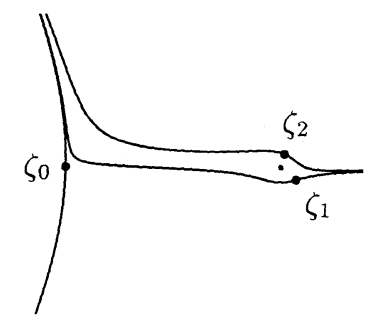

Figure 6-5

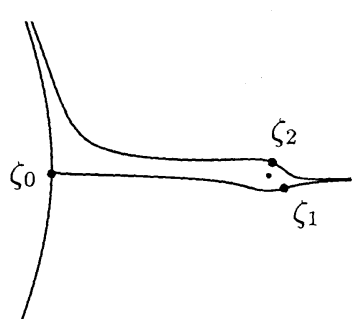

Figure 6-6

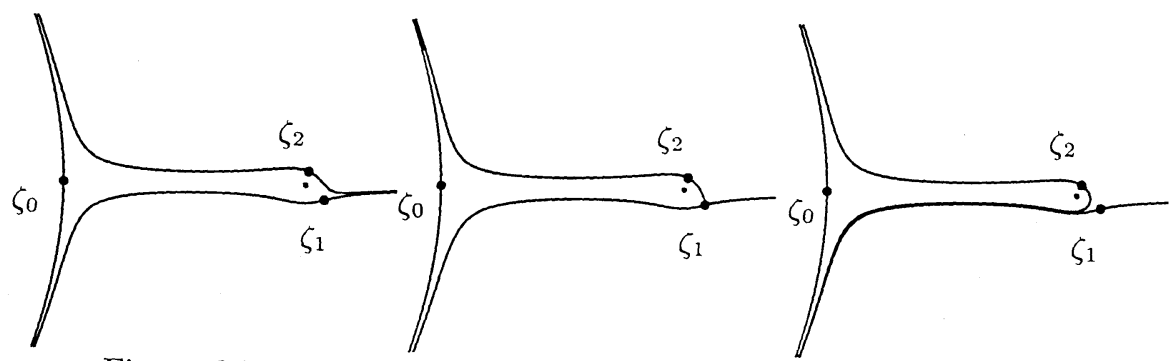

Figure 6-7

Figure 6-8

Figure 6-9 


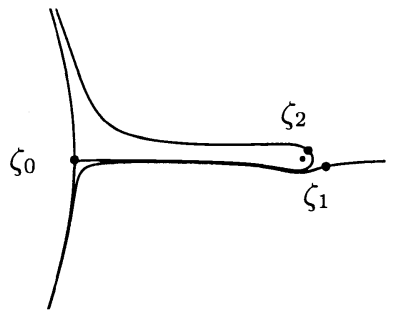

Figure 6-10

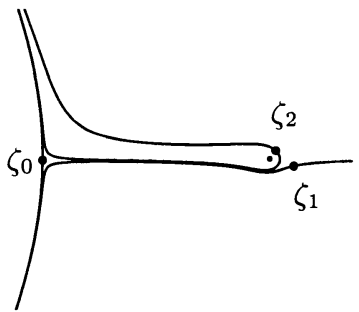

Figure 6-11

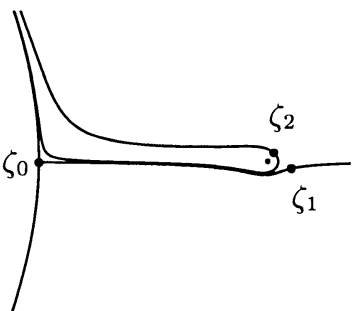

Figure 6-12

The change of configuration obtained here is completely the same with that in Example 2.1, which supports the validity of our Ansatz near ordered crossing points of an ordinary Stokes curve with a new Stokes curve.

3. Acknowledgment. This work was completed when the authors stayed at Brunel University under the support of Japan Society for the Promotion of Science. The authors sincerely thank Dr. C. J. Howls and his colleagues at Brunel University for their hospitality. The authors also thank Dr. T. Koike for his great help in including most of the pictures in this paper into a $\mathrm{T}_{\mathrm{E}} \mathrm{X}$ file.

\section{REFERENCES}

[AKT] T. AOKI, T. KAWAI AND Y. TAKEI, New turning points in the exact WKB analysis for higherorder ordinary differential equations, Analyse algébrique des perturbations singulières, I, Méthodes résurgentes, Hermann, 1994, pp. 69-84.

[BH] M. V. Berry ANd C. J. Howls, Hyperasymptotics for integrals with saddles, Proc. Roy. Soc. Lond. A, 434 (1991), pp. 657-675.

[BNR] H. L. Berk, W. M. Nevins And K. V. Roberts, New Stokes' line in WKB theory, J. Math. Phys., 23 (1982), pp. 988-1002.

[CH] C. E. Carroll and F. T. Hioe, Transition probabilities for the three-level Landau-Zener model, J. Phys. A, 19 (1986), pp. 2061-2073.

[DDP] E. Delabaere, H. Dillinger et F. Pham, Résurgence de Voros et périodes des courbes hyperelliptiques, Ann. Inst. Fourier (Grenoble), 43 (1993), pp. 163-199.

[H] C. J. Howls, An introduction to hyperasymptotics using Borel-Laplace transforms, RIMS Kôkyûroku, 968 (1996), pp. 31-48.

[SKK] M. SATo, T. KAWAi AND M. KASHIWARA, Microfunctions and pseudo-differential equations, Lect. Notes in Math., 287, Springer, 1973, pp. 265-529.

[U1] K. UchiYAma, On examples of Voros analysis in complex WKB theory, Analyse algébrique des perturbations singulières, I, Méthodes résurgentes, Hermann, 1994, pp. 115-134.

[U2] - Graphic illustration of "double Stokes phenomena”, RIMS Kôkyûroku, 968 (1996), pp. 104-109.

[V] A. Voros, The return of the quartic oscillator. The complex WKB method, Ann. Inst. Henri Poincaré, 39 (1983), pp. 211-338. 